Nervenarzt 2014 $\cdot 85: 803-804$

DOI 10.1007/s00115-013-3991-5

Online publiziert: 8. Juni 2014

๑) Springer-Verlag Berlin Heidelberg 2014
G. Laux ${ }^{1,2} \cdot$ B. Widder ${ }^{3}$

${ }^{1}$ Institut für Psychologische Medizin, Haag i.OB

${ }^{2}$ Klinik und Poliklinik für Psychiatrie und Psychotherapie,

Klinikum der Ludwig-Maximilians-Universität München

${ }^{3}$ Klinik für Neurologie und Neurologische Rehabilitation, Bezirkskrankenhaus Günzburg

\title{
Fahreignung bei psychischen und neurologischen Erkrankungen
}

Dem Thema Fahrtauglichkeit kommt in unserer modernen Gesellschaft mehr denn je höchste Bedeutung zu. Autofahren sichert Mobilität, die Voraussetzung für viele berufliche Tätigkeiten sowie für die Bewältigung und Gestaltung des gesellschaftlich-privaten Alltagslebens ist. Basis der Beurteilung ist die Fahrerlaubnisverordnung $(\mathrm{FeV})$, ergänzt durch die zuletzt zum 01.05.2014 aktualisierten Begutachtungsleitlinien zur Kraftfahreignung, die einen Rahmen für die Aufklärung von Patienten über die Fahreignung sowie ggf. für deren Begutachtung geben [2]. Diese gliedern sich in einen allgemeinen sowie einen speziellen Teil mit Ausführungen zu Krankheitsbildern. Unsere Fachgebiete betreffen folgende Abschnitte: Krankheiten des Nervensystems, psychische Störungen, Alkohol, Betäubungsund Arzneimittel. Die Darstellung orientiert sich leider nicht an den Vorgaben der ICD-10 und entspricht hinsichtlich neurologischer und psychischer Störungen nicht unserem fachärztlichen Vorgehen. Beispielsweise werden Psychopharmaka nur kurz im Abschnitt „Betäubungs- und Arzneimittel“ als „psychoaktive Stoffe “ erwähnt, differenzierte Aussagen finden sich nicht. Vor diesem Hintergrund erscheint uns dieses Schwerpunktheft für die Kollegen unserer Fachgebiete dringlich geboten.

Die Rahmenbedingungen der Fahreignungsdiagnostik implizieren eine entscheidungsorientierte Diagnostik. Wesentliche Elemente sind

- die Überprüfung der Verwertbarkeit von Angaben und Befunden,
- der Ausschluss aktueller Beeinträchtigungen der Fahrfähigkeit und

- die Bewertung eines Veränderungsprozesses [5].

Die neue Auflage der Beurteilungskriterien, herausgegeben von der Deutschen Gesellschaft für Verkehrspsychologie und der Deutschen Gesellschaft für Verkehrsmedizin, legt die Urteilsbildung in der Fahreignungsbegutachtung ausführlich dar. Der typische Ablauf ist eine Aktenund Vorgeschichtsanalyse, das Gutachten selbst muss nachvollziehbar, nachprüfbar und verständlich sein. In diesem Buch findet sich auch ein Schema zum Gutachtenaufbau [6].

\section{D) Die Begutachtung der Fahreignung erfolgt interdisziplinär}

Prinzipiell erfolgt die Begutachtung der Fahreignung interdisziplinär, d. h. es findet eine medizinisch-psychologische Untersuchung statt. Zu den Untersuchungsmitteln zählen auch chemischtoxikologische Untersuchungen insbesondere bei den Fragestellungen Alkohol, Drogen und/oder Medikamentenmissbrauch. Ein relevanter Aspekt ist hier z. B. die Nachweisbarkeitsdauer von Drogen im Urin oder in Haaren. Die psychologischen Testverfahren beinhalten folgende fünf Leistungsbereiche: Belastbarkeit, Orientierungsleistung, Konzentrationsfähigkeit, Aufmerksamkeitsleistung und Reaktionsfähigkeit. Psychologische
Testbatterien werden in Deutschland relativ wenig im Rahmen der medizinischpsychologischen Untersuchung eingesetzt, bei älteren Kraftfahrern wird eine praktische Fahrverhaltensprobe empfohlen. Die medizinische Untersuchung beinhaltet ärztliche Untersuchungsbefunde durch den für die Fragestellung zuständigen Facharzt mit verkehrsmedizinischer Qualifikation. Der Mindestuntersuchungsumfang der medizinischen Untersuchung ist festgelegt $[3,5,6]$. Im Handbuch für Verkehrsmedizin sind Ausführungen zur Fahreignung und Fahrsicherheit anschaulich und praxisgerecht für alle Medizingebiete niedergelegt [4].

Die vorliegende Ausgabe von Der Nervenarzt umfasst insgesamt sieben Artikel zum Leitthema „Fahreignung“:

Hinsichtlich allgemeiner Fragen zur Fahreignung und insbesondere zu formal-juristischen Fragen besteht erfahrungsgemäß große Unsicherheit. Das Einführungskapitel gibt hierzu eine konzis-verständliche Übersicht. Wesentlich erscheint dabei auch die Unterscheidung zwischen der Aufklärung im Rahmen der Patientenversorgung und der verkehrsmedizinischen Begutachtung auf Anordnung der Fahrerlaubnisbehörde.

Dem Thema Demenz und Autofahren kommt angesichts der demographischen Entwicklung wachsende Bedeutung zu. Gerade hier bedarf es einer differenzierten individuellen Diagnostik so sollten z. B. Patienten mit frontotemporaler Demenz frühzeitig das Autofahren einstellen. Mittels Screening-Tests kann eine Abschätzung der funktionalen 
Demenzbeeinträchtigungen bezüglich visuell-räumlicher Fähigkeiten, Exekutivfunktionen und der Aufmerksamkeitsleistung erfolgen.

Alkoholbezogene Fragestellungen dominieren in der Gutachtenpraxis eindeutig. Der zugehörige Beitrag gibt die Grundzüge der Begutachtung hierzu anschaulich wieder. Bemerkenswert ist, dass substituierte Opiatabhängige hinsichtlich fahrtauglichkeitsrelevanter kognitiver Funktionen zumeist kaum beeinträchtigt sind, wenn keine anderen gesundheitlichen Einschränkungen vorliegen.

Bei akut an Schizophrenie Erkrankten besteht keine Fahrtauglichkeit. Zur Wiedererlangung der Fahrerlaubnis sind unter fachärztlichen Kontrollen bei Ersterkrankten die Freiheit von realitätsbeeinträchtigenden Symptomen, bei rezidivierendem Verlauf mehrjährige Intervalluntersuchungen je nach individuellem Verlauf erforderlich.

Die Datenlage bezüglich Fahrtauglichkeit und Unfallrisiken depressiver Patienten ist spärlich und methodologisch schwierig. Bei akuten schweren Depressionen besteht ebenso wie in manischen Phasen keine Fahrtauglichkeit, nach Remission kann in der Regel wieder von Fahreignung ausgegangen werden.

\section{》) Erfolgreich behandelte depressive Patienten zeigen günstigere Testresultate}

Studien zur Fahrtauglichkeit unter Psychopharmaka wurden überwiegend an jungen gesunden Probanden durchgeführt, es liegen jetzt aber auch Ergebnisse von Patienten-Samples vor. Nach negativen Akuteffekten zeigen erfolgreich mit Antidepressiva behandelte Patienten günstigere Testresultate als unbehandelte Depressive. Patienten lassen unter neueren selektiven Antidepressiva ebenso wie unter sog. atypischen Antipsychotika zumeist bessere Ergebnisse erkennen als unter älteren Präparaten. BenzodiazepinTranquilizer und -Hypnotika erhöhen in Abhängigkeit von Dosierung, Einnahmezeitpunkt und Eliminationshalbwertszeit eindeutig das Verkehrsunfallrisiko, ebenso ein Teil der freiverkäuflichen Antihistaminika. Bei jungen ADHS-Patienten be- wirkt Methylphenidat eindeutig günstige Effekte auf die Fahrtauglichkeit.

Für den Bereich der neurologischen Erkrankungen wurden bewusst drei häufige Krankheitsbilder ausgewählt, für die sich in den bisherigen Begutachtungsleitlinien zur Kraftfahreignung entweder keine oder wenig brauchbare Angaben fanden. Die Problematik epileptischer Anfälle und Epilepsien wurde demgegenüber in den Leitlinien bereits 2009 wesentlich überarbeitet und entspricht dem aktuellen Stand der Wissenschaft, Gleiches gilt für Parkinson-Erkrankungen, für die unlängst aus neurologischer Sicht detaillierte Kriterien publiziert wurden [1].

Die Multiple Sklerose taucht in der $\mathrm{FeV}$ und den Begutachtungsleitlinien nur am Rande bei den Rückenmarkserkrankungen auf, was dem vielfältigen Erscheinungsbild der Erkrankung keinesfalls gerecht wird. Zu beurteilen sind vielmehr sowohl das kognitive als auch das körperliche Leistungsniveau einschließlich der Frage des bestehenden Störungsbewusstseins. Darüber hinaus gilt es, Kompensationsmöglichkeiten abzuklären, um die Mobilität der Betroffenen möglichst lange zu erhalten.

Bei Hirndurchblutungsstörungen kommt es insbesondere bei der Frage nach der Fahreignung von Berufskraftfahrern (Gruppe 2) immer wieder zu Problemen, da die Begutachtungsleitlinien recht pauschal eine Eignung für das Führen von Fahrzeugen bei kreislaufabhängigen Störungen der Hirntätigkeit verneinen. Auf dem Boden einer detaillierten literaturbasierten Gefährdungsanalyse werden in der vorliegenden Arbeit erstmals praktisch verwendbare Vorschläge für eine differenzierte Einschätzung der Fahreignung nach Schlaganfällen und insbesondere auch transitorisch ischämischen Attacken vorgelegt.

Ein bislang wenig beachtetes Gebiet war die Fahreignung von Patienten mit Schwindel und Gleichgewichtsstörungen. Aus neurologischer Sicht werden die brandneuen Leitlinien zur Begutachtung von Gleichgewichtsstörungen vorgestellt, die nach Zustimmung durch den Bundesrat zum 01.05.2014 Eingang in die jüngste Version der Begutachtungsleitlinien zur Kraftfahreignung fanden [2].
Mit diesen Beiträgen möchten wir allen Kollegen in Klinik und Praxis eine rasche, übersichtliche Orientierung und Hilfestellung zu Entscheidungen hinsichtlich der Fahrtauglichkeit ihrer Patienten ermöglichen. Gleichzeitig möchten wir dazu anregen, bei der Erhebung fundierter empirischer Daten und praxisrelevanter Forschungsansätze mitzuwirken.

Mit freundlichen kollegialen Grüßen

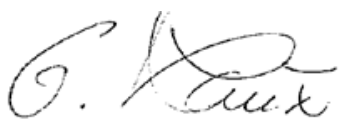

Prof. Dr. Gerd Laux

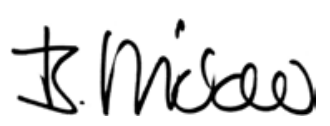

Prof. Dr. Dr. Bernhard Widder

\section{Korrespondenzadressen}

Prof. Dr. G. Laux

Institut für Psychologische Medizin

Oberwallnerweg 7, 83527 Haag i.OB

ipm@ipm-laux.de

\section{Prof. Dr. Dr. B. Widder}

Klinik für Neurologie und Neurologische Rehabilitation, Bezirkskrankenhaus Günzburg Ludwig-Heilmeyer-Straße 2, 89312 Günzburg bernhard.widder@bkh-guenzburg.de

Interessenkonflikt. G. Laux und B. Widder geben an, dass kein Interessenkonflikt besteht.

\section{Literatur}

1. Buhmann C, Gerloff C (2013) Autofahren bei Morbus Parkinson. Akt Neurol 40: 315-320

2. Bundesanstalt für Straßenwesen (2014) Begutachtungsleitlinien für die Kraftfahreignung. www.bast.de

3. Golka K, Hengstler JG, Letzel St, Nowak D (Hrsg) (2011) Verkehrsmedizin - arbeitsmedizinische Aspekte. Orientierungshilfe für Praxis, Klinik und Betrieb. Ecomed Medizin, Heidelberg

4. Madea B, Musshoff F, Berghaus G (Hrsg) (2012) Verkehrsmedizin - Fahreignung, Fahrsicherheit, Unfallrekonstruktion. 2.Aufl. DÄV, Köln

5. Schubert W, Mattern R (Hrsg) (2009) Urteilsbildung in der medizinisch-psychologischen Fahreignungsdiagnostik - Beurteilungskriterien. 2. Aufl. Kirschbaum, Bonn

6. Schubert W, Dittmann V, Brenner-Hartmann J (Hrsg) (2013) Urteilsbildung in der Fahreignungsbegutachtung. Beurteilungskriterien. 3.Aufl. Kirschbaum, Bonn 